Reprod. Nutr. Dévelop., 1980, 20 (5 B), 1723-1730.

\title{
Étude comparative de la digestion d'un aliment complet chez le poney et le lapin
}

\author{
par R. WOLTER, F. NOUWAKPO, Andrée DURIX \\ Chaire de Nutrition et d'Alimentation \\ Ecole Nationale Vétérinaire de Lyon \\ Marcy L'Efoile, 69250 Charbonnières les Bains, France.
}

Summary. Comparative digestibility of a complete pelleted diet in ponies and rabbits.

A complete pelleted diet (table 1) containing 11 p. 100 of crude protein and 17 p. 100 of cellulose (ADF-lignine according to the method of Van Soest) was distributed to ponies and rabbits. Total digestibilities, partial cumulative digestibilities in different compartments of the digestive tract (stomach, small intestine, caecum, proximal and distal colon) and changes in biochemical composition, measured by $\mathrm{pH}, \mathrm{VFA}$, lactic acid and ammonia concentrations, were compared.

Total digestibilities (table 2). There was no significant difference in dry matter between the two species. Crude protein digestibility was higher in ponies, but a greater difference was observed for crude fiber digestibility : digestibility in the rabbit was 60 p. 100 of that in the pony. Starch was entirely digested in ponies and rabbits.

Partial digestibilities (table 3). The dry matter content was the same in the stomach, the different parts of the small intestine and the caecum of both species. Starch was rapidly digested, but crude protein digestibility was highly negative in the stomach, small intestine and caecum of both species.

Changes in biochemical composition (table 4). VFA concentrations were higher in the caecum and colon of ponies but molar percentages of acetic, propionic and butyric acids were very comparable (fig. 1) ; L-lactic acid concentration was higher in the first part of the digestive tract of ponies and ammonia concentration was lower.

Les équidés et les léporidés sont des monogastriques herbivores qui présentent des similitudes anatomiques et physiologiques de leur tractus digestif, avec, en particulier, un assez large développement du gros intestin où se déroule une importante activité microbienne permettant nolamment une utile digestion des parois végétales. Par contre, les premiers se distinguent des seconds par le faible volume de leur estomac, par leur absence de cæcotrophie, mais par leur plus grande aptitude à digérer la cellulose comme cela a été démontré avec des rations à base de foin (Slade et Hintz, 1969) ou de maïs-fourrage (Schurg et al., 1977).

Pour mieux comparer l'efficacité digestive des équidés et des léporidés, nous avons déterminé chez des poneys et des lapins recevant un même aliment complet, d'une part les digestibilités apparentes globales, d'autre part les digestibilités partielles qui sont mesurées au niveau des différents segments du tube digestif chez des ani- 
maux abottus; en parallèle, nous avons également suivi l'évolution de quelques paramètres biochimiques du contenu intestinal : $\mathrm{pH}$, acides gras volatils, acide lactique, ammoniac.

\section{Matériel et méthodes.}

1. - Digestibilités totales.

a) Animaux : Six poneys adultes de $180 \mathrm{~kg}$ de poids moyen et douze lapins « Fauve de Bourgogne », de $3 \mathrm{~kg}$ de poids moyen, sont placés en cages à métabolisme.

b) Aliment : les animaux reçoivent un aliment complet, dont la composition est rapportée au tableau 1, et auquel a été incorporé de l'oxyde de chrome au taux de 0,2 p. 100.

TABLEAU 1

Composition de l'aliment complet

\begin{tabular}{|c|c|c|}
\hline p. 100 & Poney & Lapin \\
\hline 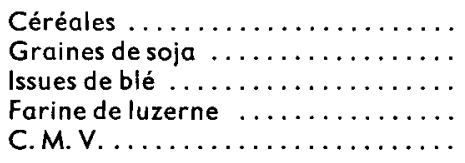 & $\begin{array}{r}40 \\
15 \\
42 \\
3\end{array}$ & $\begin{array}{c}34,7 \\
5,3 \\
15 \\
42 \\
2,6\end{array}$ \\
\hline \multicolumn{3}{|l|}{ P. 100 de la matière sèche } \\
\hline 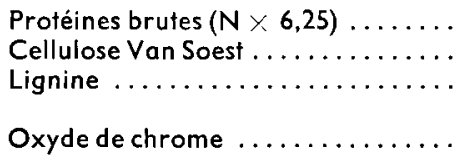 & $\begin{array}{c}12,8 \\
18,2 \\
4,7 \\
30,7 \\
0,20\end{array}$ & $\begin{array}{c}11,2 \\
17,3 \\
3,6 \\
38,0 \\
0,28\end{array}$ \\
\hline
\end{tabular}

Les rations sont ajustées à $60 \mathrm{~g} / \mathrm{kg} \mathrm{PV}^{\mathrm{75}} / \mathrm{jour}$, correspondant à peu près aux besoins d'entretien : chez les poneys, elles sont distribuées à raison de $3 \mathrm{~kg} / \mathrm{Al} / \mathrm{jour}$, en 2 repas, à $8 \mathrm{~h}$ ef à $17 \mathrm{~h}$; les lapins consomment $140 \mathrm{~g} / \mathrm{Al} / \mathrm{jour}$, en 1 seul repas à $9 \mathrm{~h}$, et pratiquent librement la cæcotrophie.

Après quatre semaines d'adaptation au régime, les fèces sont récoltées intégralement pendant six jours consécutifs.

\section{Etude des contenus intestinaux.}

Quatre poneys, destinés à l'enseignement pratique d'anatomie sont adaptés pendant 4 semaines à l'aliment complet ( $3 \mathrm{~kg} / \mathrm{Al} / \mathrm{jour}$ ) qu'ils reçoivent en 1 seule fois, le matin à $9 \mathrm{~h}$. L'abattage est réalisé par saignée après anesthésie générale, trois heures après la distribulion du repas. L'éviscération a lieu dans l'heure qui suit, de telle sorte qu'il s'écoule presque $4 \mathrm{~h}$ entre le repas et le prélèvement des contenus digestifs. Le moment du prélèvement a été déterminé à la suite des résultats de nos études antérieures de la vitesse du transit digestif chez des poneys (données non publiées) de manière à ce qu'une fraction de la ration aif déjà affeint le cæcum. 
Par ailleurs 10 lapins, préalablement utilisés pour les mesures de digestibilité totale, subissent une dislocation cervicale, puis sont saignés et éviscérés. Les contenus gastrointestinaux sont prélevés dans les $10 \mathrm{~min}$ qui suivent l'abattage. Celui-ci intervient $4 \mathrm{~h}$ après la distribution du repas, d'une part pour se placer dans les mêmes conditions que pour les poneys, ef d'autre part de manière à se situer en dehors des périodes de cæcotrophie. En effet, Fioramonti et Ruckebusch (1976) ont mis en évidence que la cæcotrophie est déclenchée 6 à $8 \mathrm{~h}$ après la prise alimentaire.

Les sites de prélèvement sont donnés dans le tableau 2.

TABLEAU 2

Sites de prélèvement

\begin{tabular}{|c|c|c|c|c|c|c|c|}
\hline Poney & Estomac & $\begin{array}{l}\text { 1er tiers } \\
\text { Int. grêle }\end{array}$ & $\begin{array}{c}2^{\mathrm{e}} \text { tiers } \\
\text { Int. grêle }\end{array}$ & $\begin{array}{l}3^{e} \text { tiers } \\
\text { Int. grêle }\end{array}$ & Cæcum & $\begin{array}{l}\text { Côlon } \\
\text { dorsal }\end{array}$ & $\begin{array}{l}\text { Côlon } \\
\text { ventral }\end{array}$ \\
\hline Lapin & Estomac & Duodenum & Jejunc & o-iléon & Cxcum & $\begin{array}{l}\text { Côlon } \\
\text { proximal }\end{array}$ & $\begin{array}{l}\text { Côlon } \\
\text { distal }\end{array}$ \\
\hline
\end{tabular}

Le contenu total de chaque compartiment est pesé ; l'humidité est déterminée sur une fraction aliquote de ce contenu, le résidu sec étant stocké en vue des analyses permettant le calcul des digestibilités cumulées ; une autre fraction est destinée aux mesures des paramètres biochimiques.

\section{Techniques analytiques.}

a) Digestibilité : les coefficients d'utilisation digestive (CUD) totale ont été déterminés par la méthode de récolte intégrale des fèces, alors que les digestibilités cumulées estimées le long du tube digestif ont été calculées à l'aide du marqueur incorporé à l'aliment (oxyde de chrome). Les différents éléments : matières azotées totales, cellulose, lignine, amidon, minéraux totaux, calcium, phosphore, oxyde de chrome, ont été dosés selon les méthodes habituellement appliquées au laboratoire (Wolter, Durix et Letourneau, 1976).

b) Paramètres biochimiques: Après homogénéisation du prélèvement à la spatule, le pH est immédiatement mesuré à l'aide d'un $\mathrm{pH}$-mètre ; puis 2 à $10 \mathrm{~g}$ d'échantillon sont dilués dans de l'eau distillée en quantité connue, en fonction de la consistance du prélèvement. La suspension est préparée en vue des dosages des acides gras (AGV), des acides $D$ et L-lactiques, de l'ammoniac, puis stockés à $-20^{\circ} \mathrm{C}$. Les analyses sont effectuées selon des techniques précédemment décrites (Wolter et al., 1978).

\section{Résultats et discussion.}

1. - Digestibilités totales. Le tableau 3 récapitule les digestibilités totales chez le poney et le lapin. II n'apparaît pas de différence entre les deux espèces, quant à la digestibilité de la matière sèche, alors que les matières azotées sont significativement moins digérées par le lapin $(P<0,05)$. 
TABLEAU 3

Digestibilité totale comparée chez le poney ef le lapin (moyenne : 6 poneys et 10 lapins)

p. 100

Poney

Lapin

Matière sèche .................

Protéines brutes $(\mathrm{N} \times 6,25) \ldots \ldots$

Cellulose Van Soest . ..........

Amidon .................

\author{
$63,39 \pm 1,84$ \\ $74,05 \pm 1,84 a *$ \\ $51,22 \pm 4,03\left({ }^{1}\right) * c$ \\ $98,02 \pm 0,24$
}

$61,84 \pm 2,32$

$65,93 \pm 3,28 b$

$25,46 \pm 4,63$ d

$96,77 \pm 0,55$

* Les résultats affectés de deux leftres différentes diffèrent significativement $(P<0,05)$.
(1) Moyenne effectuée sur 3 poneys.

Surtout, nos résultats montrent bien que la digestibilité de la cellulose, dosée selon la méthode de Van Soest (ADF — lignine) est nettement plus faible, de l'ordre de moitié, chez le lapin par rapport au poney : environ 25 p. 100 au lieu de 51 p. 100 dans le cas présent. Ils confirment pleinement ceux obtenus, lors d'une même comparaison, par Slade ef Hintz (1969) avec un régime plus fibreux à base de luzerne (18 p. 100 contre 36 p. 100), ainsi que par Schurg et al. (1977) avec du maïs-fourrage (37 p. 100 et 69 p. 100). De même, à partir d'essais conduits exclusivement sur lapin, Candau, Bertrand ef Fioramonti (1978) estiment à 22 p. 100 la digestibilité de cette même cellulose apportée en majorité par de la luzerne. Par ailleurs, notons que les teneurs en amidon, des divers régimes comparés chez le poney et chez le lapin, ne semblent pas influencer nettement la digestibilité des parois végétales chez ces animaux.

L'amidon est très bien utilisé par les 2 espèces puisque sa digestibilité totale atteint 98 p. 100 chez le poney ef 96 p. 100 chez le lapin.

2. Digestibilités partielles et évolution des paramètres biochimiques.

a) Digestibilités partielles.

Le tableau 4 rapporte l'évolution du taux de matière sèche ainsi que les coefficients d'utilisation digestive partielle, tout au long du tube gastro-intestinal.

Les taux de matière sèche et la digestibilité de celle-ci évoluent de manière similaire chez le poney et le lapin jusqu'au cæcum, les digestibilités apparentes parfois négatives résultant des abondantes sécrétions digestives. Ces résultats suggèrent que le transit el l'absorption de l'eau se déroulent de manière identique chez les 2 espèces. Cependant à partir du cæcum, la teneur en matière sèche des digesta est plus faible chez le poney; comme chez celui-ci la digestibilité partielle atteinte à ce niveau du tube digestif n'est pas plus élevée que chez le lapin, il faut admettre une plus rapide absorption des produits d'hydrolyse.

L'amidon est digéré pour sa grande majorité dès l'intestin grêle puisqu'il ne se retrouve au niveau du cæcum que pour 5 p. $100 \mathrm{chez}$ le poney, et pour 15 p. $100 \mathrm{chez}$ le lapin. Le concernant, la digestion enzymatique prime donc très largement la fermentation microbienne chez ces monogastriques herbivores qui apparaissent à cet égard beaucoup plus éloignés des ruminants que du porc. En effet, chez celui-ci, Keys et De Barthe (1974) ont mis en évidence que la presque totalité de l'amidon a disparu dans l'intestin grêle. 


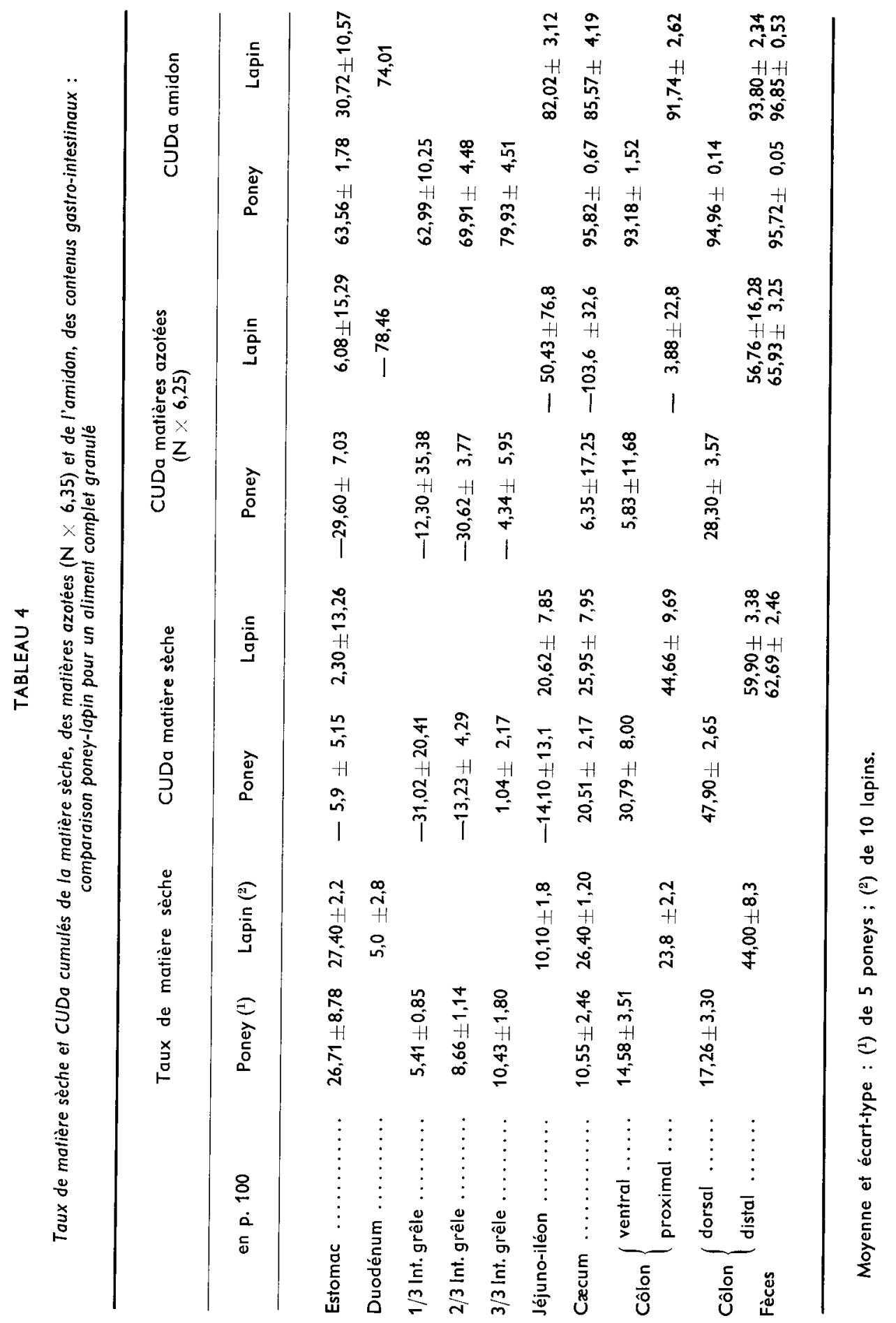




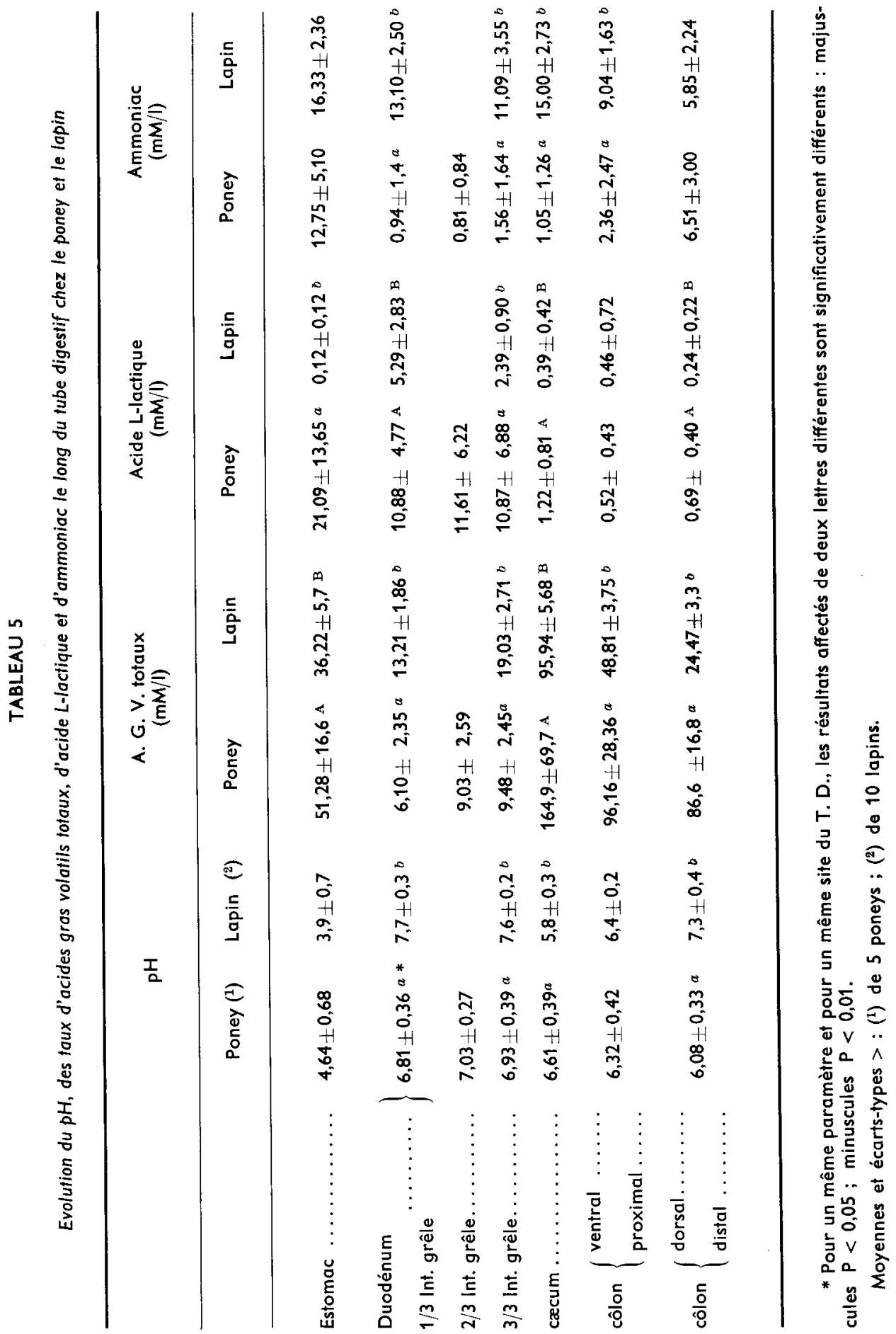


La digestibilité des matières azotées reste fortement négative, particulièrement chez le lapin. L'interprétation de ces CUD reste délicate du fait de l'irruption massive d'azote endogène et, en outre, de la cæcotrophie chez le lapin. Les faibles valeurs observées chez le poney peuvent provenir d'une sous-estimation de la digestibilité calculée avec le marqueur oxyde de chrome; nous avons en effet montré sur des poneys porteurs de fistules cæcales que la digestibilité à ce niveau du tube digestif atteint près de 53 p. 100 (Wolter, Durix et Letourneau, 1976) ; sans doute faut-il supposer que l'oxyde de chrome, incorporé sous forme de poudre dans l'aliment composé granulé, se "démélange » au cours du transit digestif et constitue donc un marqueur médiocre pour ce type d'étude ; des essais menés avec d'autres marqueurs devraient fournir des précisions, à ce sujet.

b) Paramètres biochimiques.

- Chez les 2 espèces, le $\mathrm{pH}$ est minimum dans l'estomac, sans doute pour une très grande part, sous l'influence de la sécrétion chlorhydrique; il s'abaisse de nouveau quelque peu au niveau du gros intestin, parallèlement à l'accumulation des acides gras volatils (tabl. 5).

- Ces derniers sont déjà en quantités notables dans l'estomac, particulièrement chez le poney, vraisemblablement à cause d'une attaque microbienne très précoce des glucides facilement fermentescibles. Ils se concentrent surtout dans le gros intestin, spécialement dans le cæcum, et de nouveau davantage chez le poney qui manifeste ainsi sa plus forte activité cellulolytique.

- Les proportions des différents acides gras volatils dans le mélange total évolue de façon comparable chez les 2 espèces, tout au long de leur tube digestif, avec une nette prédominance de l'acide propionique dans l'estomac et l'intestin grêle, puis au contraire une forte majorité d'acide acétique dans le gros intestin (fig. 1). Cette production accrue d'acide propionique dans les segments antérieurs du tube digestif

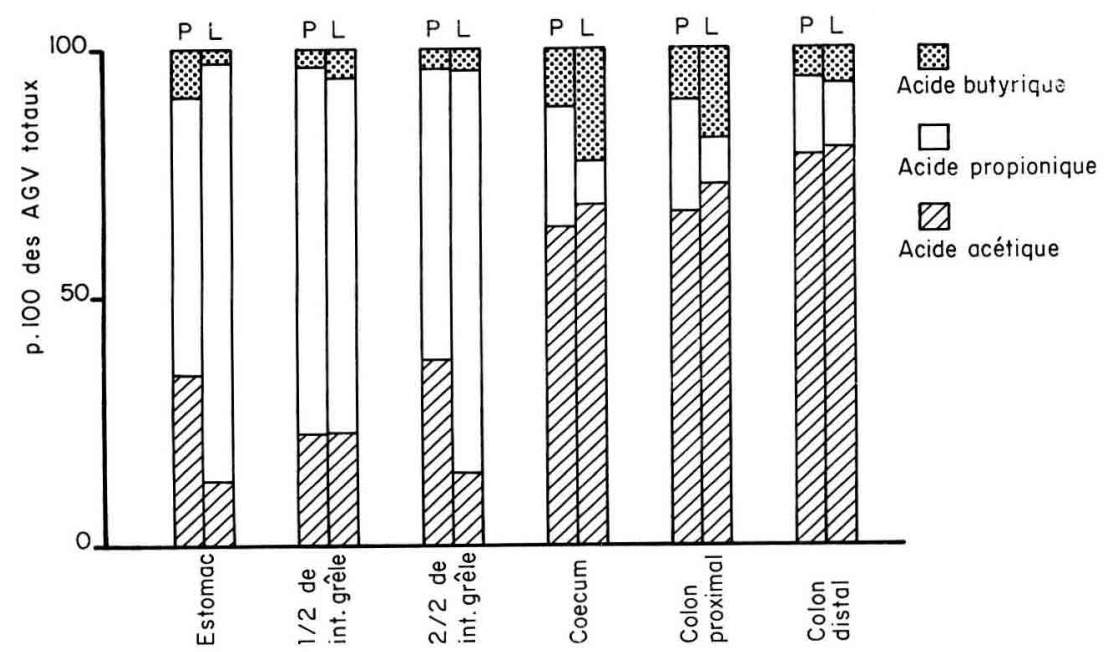

FIG. 1. - Proportions relatives d'acides gras volatils, le long du lube digestif = poney/lapin (p. 100 des AGV totaux). 
doit être en rapport avec la richesse relative, du régime utilisé, en amidon ; elle fut déjà bien observée chez le lapin aussi bien par Ruckebusch et Fioramonti (1976), que par Bonnafous et Raynaud (1978) ; par contre, elle n'apparaît pas chez le poney recevant un régime à base de luzerne, qui privilégie la fermentation acétique (Argenzio, Southworth et Stevens, 1974).

- La concentration en acide L-lactique est relativement élevée, significativement plus chez le poney que chez le lapin, dans la première partie du tube digestif, sans doute parce que la microflore lactique profite, surtout au niveau de l'estomac, d'un $\mathrm{pH}$ suffisamment bas et d'un substrat bien pourvu en glucides facilement fermentescibles. De même, Argenzio, Southworth et Stevens (1974) avaient constaté que cette production stomacale d'acide lactique est accrue avec des régimes amylacés alors qu'elle est pratiquement annulée lors de consommation exclusive de fourrages.

- L'ammoniac apparaît beaucoup plus abondant dans le contenu digestif du lapin jusqu'au côlon proximal. Par contre chez le poney, son taux reste assez bas jusqu'au début du gros intestin et la chute brutale de concentration entre l'estomac ef le début de l'intestin grêle laisse supposer une résorption intense dans cette zone. Chez le lapin, l'accumulation relative d'ammoniac pourrait provenir soit d'une moindre absorption dans les sites qui précèdent le côlon proximal soit d'une libération accrue à partir des microorganismes d'origine cæcotrophique.

Ainsi, l'utilisation digestive d'un aliment complet se différencie chez le poney et le lapin, d'une part au niveau de la digestion des matières azotées de mode plus complexe chez le lapin par suite du comportement cæcotrophique de cefte espèce, d'autre part et surtout par la meilleure digestion de la cellulose brute chez le poney.

journées Ingestion-Digestion-Absorption de l'Association française de Nutrition, Paris, 15-16 novembre 1979.

\section{Références}

ARGENZIO R. A., SOUTHWORTH M., STEVENS C. E., 1974. Site of organic production and adsorption in the equine gastrointestinal tract. Am. J. Physiol., 226, 1043-1050.

BONNAFOUS R., RAYNAUD P., 1978. Etude sur la concentration des acides gras volatils du matériel et des acides gras volatils et corps cétoniques plasmatiques au niveau des veines du gros intestin chez le lapin domestique. $2^{\mathrm{e}}$ Journ. Rech. cunic. en France, Toulouse.

CANDAU M., BERTRAND B., FIORAMONTI J., 1978. Variation de la digestibilité des constituants de la ration chez le lapin. C. R. Soc. Biol., 172, 554-559.

FIORAMONTI J., RUCKEBUSCH Y,, 1976. La motricité cæcale chez le lapin. III. - Dualité de l'excréfion fécale. Ann. Rech. vét., 7, 281-295.

KEYS J. E., DE BARTHE J. V., 1974. Site and extent of carbohydrate, dry matter, energy and protein digestion, and the rate of passage of grain diets in swine. J. Anim. Sci., 39, 57-62.

RUCKEBUSCH F., FIORAMONTI J., 1976. Déterminisme de la cæcotrophie chez le lapin. Laboratoire de Physiologie, Ecole nat. vét. Toulouse.

SCHURG N. A., FREI D. L., CHEEKE P. R., HOLTAN D. W., 1977. Utilization of whole corn plant pellets by horses and rabbits. J. Anim. Sci., 45, 1317-1321.

SLADE L. M., HINTZ H. F., 1969. Comparison of digestion in horses, ponies, rabbits and guinea pigs. J. Anim. Sci., 28, 42.

WOLTER R., DURIX A., LETOURNEAU J. C., 1976. Influence du mode de présentation d'un aliment complet sur la vitesse de transit digestif et la digestibilité chez le poney. Ann. Zootech., 25, 181188.

WOLTER R., GOUY D., DURIX A., LETOURNEAU J. C., CARCELEN M., LANDREAU J., 1978. Digestibilité et activité biochimique intracæcale chez le poney recevant un même aliment complet présenté sous forme granulée, expansée ou semi-expansée. Ann. Zootech., 27, 47-60. 